\title{
NON-DEGENERATE SOLUTIONS OF BOUNDARY-VALUE PROBLEMS
}

\author{
J. Smoller*, A. Tromba $\dagger$ and A. Wasserman $\ddagger$ \\ University of Michigan, Ann Arbor, M148109, and University of California, Santa Cruz, CA95064, U.S.A.
}

(Received 7 September 1978)

Key words: Bifurcation, non-degenerate, boundary-value problem, conjugation of vector fields, covering spaces.

\section{INTRODUCTION}

CONSIDER a second-order equation of the form

$$
s u^{\prime \prime}+f(u)=0, \quad s>0, \quad a<x<b,
$$

together with homogeneous Dirichlet or Neumann boundary conditions. Suppose that $f$ is "cubic-like", i.e., $f(-A)=f(0)=f(B)=0, A, B>0$, and $f^{\prime}(0)>0$. Clearly $u \equiv 0$ is a solution, and it is also well-known that as $s \rightarrow 0$, non-constant solutions bifurcate out of the zero solution§. Our aim is to determine whether these non-constant solutions undergo bifurcations as $s \rightarrow 0$. Solutions which do not bifurcate are termed "non-degenerate". A solution $u_{s}(x)$ of $(1)$, together with the given boundary conditions, is called "strongly non-degenerate" if and only if the equation

$$
s v^{\prime \prime}+f^{\prime}\left(u_{s}(x)\right) v=0
$$

together with the same boundary conditions implies $v \equiv 0$.

A straightforward application of the implicit function theorem shows that a strongly nondegenerate solution is non-degenerate. Our object in this paper is to derive an easily checked non-degeneracy condition and to show that when our condition holds non-degenerate solutions are also strongly non-degenerate.

We consider general systems of equations in the plane, subject to linear homogeneous boundary conditions. Our technique is to rephrase the problem in a qualitative geometric way where our non-degeneracy condition is easily derivable. We shall also show that if our condition is ever violated, then we can find a boundary-value problem which admits a degenerate solution.

In the second part of the paper, we shall attack the problem by topological methods; we feel that these techniques should prove useful for other types of problems. Thus for equations of the form (1) we shall consider first the homogeneous Neumann problem and we shall explicitly construct local conjugations of the associated one-parameter family of vector fields $X_{s}=$

\footnotetext{
* Research supported by AFOSR

$\dagger$ Research supported by NSF

$\ddagger$ Research supported by NSF

$\S$ The spectrum of the linearized operator is the set $\left\{+s \lambda_{j}+f^{\prime}(0)\right\}$, where the $\lambda_{j}$ are eigenvalues of $u^{\prime \prime}$ subject to the same boundary conditions. Here $0 \geqslant \lambda_{1} \geqslant \lambda_{2} \geqslant \ldots, \lambda_{j} \rightarrow-\infty$, so that as $s \rightarrow 0$, the spectrum picks up positive elements; non-constant solutions correspond to such points.
} 
$\left(v,-s^{-1} f(u)\right)$, into each other. Since strongly non-degenerate solutions are invariant under conjugations, this will give us another approach to the problem. For other types of boundary conditions, one must "lift" the flows to the inversal covering space; we give a short description of this technique.

Our condition can be stated rather easily in terms of the corresponding vector ficld. Thus, if $X$ denotes our vector field, then our condition is that $\tilde{p}^{\mathrm{d}} X_{p} p^{t}-X_{p} \tilde{p}>0$, provided that $X$ is never radial $\ddagger$. Here $\tilde{p}$ denotes a rotation of $p \in \mathbf{R}^{2}$ by $+\pi / 2$. For the particular system (1), our condition becomes $f(u) / u>f^{\prime}(u)$.

We remark that the non-degeneracy problem arises quite naturally in the study of the stability of steady-state solutions of reaction-diffusion equations; see [1], for example.

\section{A GEOMETRIC INTERPRETATION OF NON-DEGENERACY}

In order to motivate our approach, we consider (1), and make the change of variable $y=s^{-1 / 2} x$, to get

$$
\ddot{u}+f(u)=0, \quad a / \sqrt{s}<y<b / \sqrt{s},
$$

subject to the same boundary conditions. Thus $s \rightarrow 0$ is equivalent to having the length of the interval increase. Hence, if we consider general systems of equations in $\mathbf{R}^{2}$, we can take as bifurcation parameter, the length of the interval.

Thus consider the system

$$
u^{\prime}=f(u, v), \quad v^{\prime}=g(u, v), \quad a<t<b,
$$

subject to the linear homogeneous boundary conditions

$$
\alpha_{1} u(a)+\beta_{1} v(a)=\alpha_{2} u(b)+\beta_{2} v(b)=0 .
$$

We also assume $f(0,0)=g(0,0)=0$, and let $L_{i}, i=1,2$, denote the line $\alpha_{i} u+\beta_{i} v=0$. We assume that there is a solution of (2), (3) through $p_{0} \in L_{1}$, and for $p$ near $p_{0}$, we let $\phi(p)$ denote the image under the flow of $p$ on $L_{2}$. Let $T(p)$ denote the time the orbit takes in going from $p$ to $T(p)$; thus for $p$ near $p_{0}, T(p)$ is near $T\left(p_{0}\right)$.

Lemma 1. A solution of (2)-(3) through $p_{0} \in L_{1}$ is non-degenerate if and only if $T$ is monotone in a neighborhood of $p_{0}$.

Proof. If $T$ is monotone in a neighborhood $U$ of $p_{0}$, then for each $r$ near $b-a$, there is a unique $p$ in $U$ with $T(p)=r$. Thus the solution through $p_{0}$ is non-degenerate. Conversely, if $T$ is not monotone near $p_{0}$ then there are points $p_{i}$, and $p_{i}^{\prime}$ converging to $p_{0}$ with $T\left(p_{i}\right)=T\left(p_{i}^{\prime}\right)$; hence the solution through $p_{0}$ is degenerate.

We shall use this criterion in order to derive our non-degeneracy condition. We first need some notation. For $v=(\alpha, \beta) \in \mathbf{R}^{2}$, we define $\tilde{v}=(-\beta, \alpha)$. In terms of this notation, we see that $X$ is never radial if for $p \neq 0, X_{p} \neq 0$, we have

$$
X_{p} \cdot \tilde{p} \neq 0
$$

+ The precise statement is given in Theorem 1 below.

$\ddagger$ This condition always. holds for systems (1). 
We can now state our first result.

THEOREM 1. Let $\gamma$ be a solution of (2), (3) in an open set $U$, and suppose that (4) holds in $U$. Assume that for each $q$ on $\gamma$,

$$
\tilde{q} \mathrm{~d} X_{q} q^{t}-X_{q} \cdot \tilde{q} \geqslant 0
$$

and that for some $p$ on $\gamma$

$$
\tilde{p} \mathrm{~d} X_{p} p^{t}-X_{p} \cdot \tilde{p}>0 .
$$

Then $\gamma$ is a non-degenerate solution.

Proof. Let $\sigma(p, t)$ denote the flow of (2), $p \in \mathbf{R}^{2}, t \in \mathbf{R}$. and let $\sigma_{t}$ denote the associated diffeomorphism, $p \rightarrow \sigma_{t}(p)$. Let

$$
f_{p}(t)=\mathrm{d} \sigma_{t}(p)[p] \cdot \widetilde{\sigma_{t}(p)}
$$

Here $\mathrm{d} \sigma_{t}(p)[p]$ denotes the differential of $\sigma_{t}$ evaluated at $p$ and acting on $p$. To understand the meaning of $f_{p}$, consider Fig. 1.

We think of $L_{1}$ as the "starting manifold" and $L_{2}$ as the "final manifold". Now $\sigma_{t}(s p), s \in \mathbf{R}^{1}$ is the image of the ray through $p$ so $\left.(\mathrm{d} / \mathrm{d} s) \sigma_{t}(s p)\right|_{s=1}=\mathrm{d} \sigma_{t}(p)[p]$ is the tangent vector to the image of this ray at $\sigma_{t}(p)$. Thus, if $T=b-a$, and if we show that $f_{p}(T) \neq 0$, then $\mathrm{d} \sigma_{T}(p)[p]$ is not radial so that the image of the starting manifold under the flow is transverse to the final manifold at $\sigma_{T}(p)$. Hence, if $f_{p}(T) \neq 0$, then for points on $L_{1}$ near $p$, their images in time $T$ do not lie on $L_{2}$ and so Lemma 1 implies that the solution through $p$ is non-degenerate. We proceed to show that $f_{p}(T) \neq 0$.

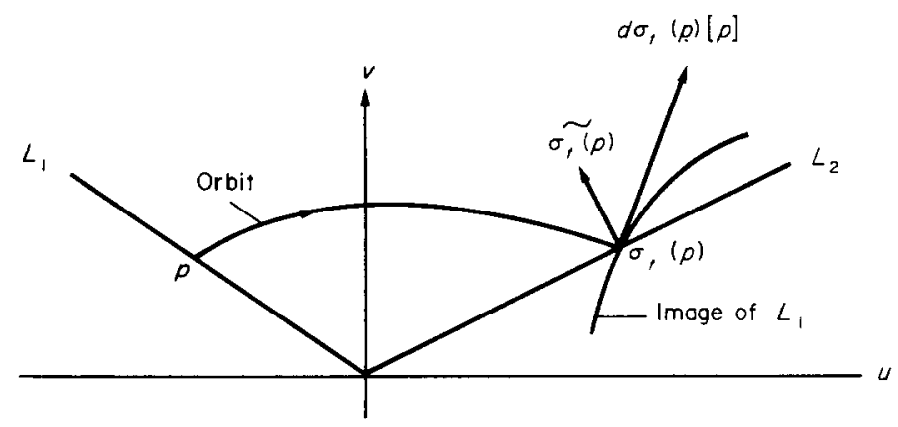

Figure 1.

Using (4), we see that at every $p \in U$, the vectors $p$ and $X_{p}$ form a basis. Let $q(t)=\sigma_{t}(p)$, $0 \leqslant t \leqslant T$, and write

$$
\mathrm{d} \sigma_{t}(p)[p]=a(t) X_{q}+b(t) q,
$$

where $a$ and $b$ are smooth scalar functions, of $p$ and $t, a(0, p)=0, b(0, p)=1$. Also, since $\mathrm{d} \sigma_{t}(p)\left[X_{p}\right]$ $=X_{q}$ and $\mathrm{d} \sigma_{t}(p)$ is an isomorphism, $b(t)>0,0 \leqslant t \leqslant T$. Now $f_{p}(t)=a(t)\left(X_{q} \cdot \tilde{q}\right)$ and since $X_{q} \cdot \tilde{q}<0$, we will be done if we show $a(T) \neq 0$.

We differentiate (7) with respect to $t$ and use the well-known differential equation satisfied by 
the space derivative of a flow $[2, \mathrm{p} .300]$, to get

$$
\begin{aligned}
\mathrm{d} X_{q} \mathrm{~d} \sigma_{t}(p)[p] & =\dot{a} X_{q}+\dot{b} q+a \mathrm{~d} X_{q}\left(X_{q}\right)+b X_{q} \\
& =a \mathrm{~d} X_{q}\left(X_{q}\right)+b \mathrm{~d} X_{q} q^{t}
\end{aligned}
$$

so that $b\left\{\tilde{q} \mathrm{~d} X_{q} q^{t}-X_{q} \cdot \tilde{q}\right\}=\dot{a} X_{q} \cdot \tilde{q}$. From (5) we see that $a(t) \leqslant 0,0 \leqslant t \leqslant T$, and hence (6) yields $a(T)<0$. The proof is complete.

We note that $a(T) \neq 0$ implies that $\partial T / \partial p \neq 0$. To see this, note that if we fix $p=p_{0}$ on the initial manifold, and let $q_{0}=\sigma_{T}\left(p_{0}\right)$, then we have $\sigma_{T\left(s p_{0}\right)}\left(s p_{0}\right)=\lambda(s) q_{0}$. Taking the derivative with respect to $s$ gives $X_{\lambda q_{0}}(\partial T / \partial s)\left(s p_{0}\right)+\mathrm{d} \sigma_{T\left(s p_{0}\right)}\left(s p_{0}\right)\left[p_{0}\right]=(\mathrm{d} \lambda / \mathrm{d} s) q_{0}$. Setting $s=1$, and using (7) gives $X_{40}(\partial T / \partial s)\left(p_{0}\right)+a X_{q_{0}}+b q_{0}=(\mathrm{d} \lambda / \mathrm{d} s) q_{0}$. If we take the inner product with $\tilde{q}_{0}$, we get $\left(X_{q 0} \cdot \tilde{q}_{0}\right)(\partial T / \partial s)+a\left(X_{q_{0}} \cdot \tilde{q}_{0}\right)=0$, so that $\left.-\mathrm{d} T(p) / \mathrm{d} p=-\mathrm{d} T\left(s p_{0}\right) /\left.\mathrm{d} s\right|_{s=1}=a(T / p), p\right)=a(T)<0$. We will use this remark in section 4 , where we shall prove that under the same hypotheses as in Theorem 1, solutions are in fact strongly non-degenerate.

Note that $a(T)<0$ implies that the time that the orbit through $p$ takes to get to $L_{2}$ increases as $p$ moves up $L_{1}$. Note too that our proof can be viewed as a technique for showing that $\partial / \partial r(\partial \theta / \partial t)>0$, where $r$ and $\theta$ denote the usual polar coordinates.

Corollary 1 . Let $\left\{X_{\theta}\right\}$ be a family of vector fields defined in an open set $U \subset \mathbf{R}^{2}$, which depend smoothly on a parameter $\theta$. Suppose that $\gamma_{0}$ is a solution of (2), (3) corresponding to $X_{\theta_{0}}$, and that (4) holds and that (5) and (6) hold along $\gamma_{0}$. Then there is a neighborhood $N$ of $\theta_{0}$ such that if $\theta \in N, X_{\theta}$ admits a unique solution which is non-degenerate.

Let $\gamma$ be any orbit satisfying $\Phi\left(P_{1}\right)>0, \Phi\left(P_{2}\right)<0, P_{1}, P_{2}$ on $\gamma$, say $P_{2}=\sigma_{T}\left(P_{1}\right), T>0$ (if $T<0$, the argument is similar). Now $a\left(P_{1}, t\right)$ has a maximum on $[0, T]$, which occurs at $t_{1} \in(0, T)$. Let $q=\sigma_{t_{1}}\left(P_{1}\right)$, then if $T-t_{1}>s>0$, we have

$$
(*) \mathrm{d} \sigma_{s+t_{1}}\left(P_{1}\right)\left[P_{1}\right]=\mathrm{d} \sigma_{s}(q)\left[\mathrm{d} \sigma_{t_{1}}\left(P_{1}\right)\left[P_{1}\right]\right]=\mathrm{d} \sigma_{s}\left[a_{1} X_{q}+b_{1} q\right]
$$

where $a_{1}=a\left(P_{1}, t_{1}\right), b_{1}=b\left(P_{1}, t_{1}\right)$. Hence

$$
\begin{aligned}
& a\left(P_{1}, t_{1}+s\right) X_{\sigma_{s}(q)}+b\left(P_{1}, t_{1}+s\right) \sigma_{s}(q)=\mathrm{d} \sigma_{s}\left(a_{1} X_{q}+b_{1} q\right) \\
& =a_{1} X_{\sigma_{s}(q)}+b_{1} \mathrm{~d} \sigma_{s}(q)[q] \\
& =a_{1} X_{\sigma_{s}(q)}+b_{1}\left(a(q, s) X_{\sigma_{s}(q)}+b(q, s) \sigma_{s}(q)\right)
\end{aligned}
$$

Hence $a\left(P_{1}, t_{1}+s\right)=a_{1}+b_{1} a(q, s)$, and so $b_{1} a(q, s) \leqslant 0$. Since $b_{1}>0$, we have $a(q, s) \leqslant 0$. Similarly, if in $\left(^{*}\right)$ we replace $t_{1}$ by $t_{1}-s$, we get $a\left(\sigma_{-s}(q), s\right) \geqslant 0$. If either of these quantities equals zero, then we're done, as we will see below. Suppose both quantities are not zero. Consider the function $A(\theta)=a\left(\sigma_{-\theta_{s}}(q),(1-\theta) s\right)$. We have $A(0)<0, A(1)>0$ so $A(\bar{\theta})=0$ for some $\bar{\theta}, 0<\bar{\theta}<1$.

Now let $P=\sigma_{-\bar{\theta}_{s}}(q)$, and let $\tilde{P}=\sigma_{\left(1-\bar{\theta}_{)_{s}}\right.}(P)$ and if $L_{1}$ is the ray from the origin through $\tilde{P}$, and $L_{2}$ is the ray from the origin through $\tilde{P}$, we see that bifurcation occurs for this boundary-value problem. Thus our condition is both necessary and sufficient for all boundary-value problems to be non-degenerate. 


\section{THE CONJUGATION OF VECTOR FIELDS}

In this section we shall demonstrate an approach to the strong non-degeneracy problem from the point of view of global analysis, and show that (local) conjugations of vector fields preserve strongly non-degenerate solutions.

Let $\left\{X_{\sigma}\right\}$ be a family of vector fields depending smoothly on a parameter $\sigma$, and let $\boldsymbol{M}$ denote the space of $H^{k}$ curves $\gamma=(u, v)$ defined on $a \leqslant t \leqslant b$ into $\mathbf{R}^{2}$ satisfying (3), and consider the operator $Y$ on $M$ into $H^{k}{ }^{1}$ defined by $Y_{\sigma}(\gamma)=\gamma^{\prime}-X_{\sigma}(\gamma)$. If $Y_{\sigma}^{*}(\gamma)$ denotes the linearization (Frechét derivative) of $Y_{\sigma}$ about $\gamma$, then $h$ is in the kernel of $Y_{\sigma}^{*}(\gamma)$ if and only if $h^{\prime}=\mathrm{d} X_{\sigma}(\gamma) h$. Thus $\gamma$ is a strongly non-degenerate solution (zero) of $Y_{a}$ if and only if $Y_{\sigma}^{*}(\gamma)$ is an isomorphism. Suppose now $X_{\sigma}$ and $X_{\tau}$ are defined on open subsets $U$ and $U^{\prime}$ of $\mathbf{R}^{2}$, respectively, and let $\phi$ be a diffeomorphism from an open subset of $U-Z\left(X_{\tau}\right)$ to $U^{\prime}$ where $Z\left(X_{\tau}\right)$ denotes the zero set of $X$. Then $\phi$ is said tolocally conjugate $X_{\sigma}$ to $X_{\tau}$ provided that there is an invariant set $V \subset U-Z\left(X_{\tau}\right)$ such that $\mathrm{d} \phi^{-1} X_{\sigma}(\phi(p))=X_{\tau}(p)$, for all $p$ in $V$. Suppose further that $\phi$ leaves $L_{i}$ invariant, $i=1,2$. Then $\phi$ takes flows $\gamma$ of $X_{\tau}$ which satisfy (3) into flows of $X_{\sigma}$ which satisfy (3), since if $\gamma^{\prime}=X_{\tau}(\gamma)$, then $\phi(\gamma)^{\prime}=\mathrm{d} \phi X_{\tau}(\gamma)=X_{\sigma}(\phi(\gamma))$. Moreover we have:

Proposition 1. Under the above assumption if $\gamma$ is a strongly non-degenerate flow of $X_{\tau}$ satisfying (3), then $\phi(\gamma)$ is a strongly non-degenerate flow of $X_{\sigma}$ satisfying (3).

Proof. An easy calculation shows $Y_{\tau}(\gamma)=\mathrm{d} \phi^{-1}(\gamma) Y_{\sigma}(\phi(\gamma))$, so

$$
\begin{aligned}
Y_{\tau}^{*}(\gamma) h & =\left(\mathrm{d} \phi^{-1}\right)^{*}\left[Y_{\sigma}(\phi(\gamma)), h\right]+\mathrm{d} \phi^{-1}\left[Y_{\sigma}^{*}(\phi(\gamma)) \mathrm{d} \phi(\gamma) h\right] \\
& =\mathrm{d} \phi^{-1}\left[Y_{\sigma}^{*}(\phi(\gamma)) \mathrm{d} \phi(\gamma) h\right],
\end{aligned}
$$

since $Y_{\sigma}(\phi(\gamma))=0$. Thus $Y_{\tau}^{*}(\gamma) h=0$ if and only if $Y_{\sigma}^{*}(\phi(\gamma)) \mathrm{d} \phi(\gamma) h=0$; hence $\phi$ induces an isomorphism. To complete the proof we must check the boundary conditions; i.e., we must show that if $h=\left(h_{1}, h_{2}\right) \in L_{i}$, then $\mathrm{d} \phi(\gamma) h \in L_{i}, i=1,2$. Assuming $\beta \neq 0$, we must show that $\mathrm{d} \phi(\gamma)$ $\left(h_{1},-\alpha / \beta h_{1}\right)^{t}$ is of the form $\left(\tilde{h}_{1},-\alpha / \beta \tilde{h}_{1}\right)$. Since $L\left(=L_{i}\right)$ is invariant, if $\phi\left(z_{1}, z_{2}\right)=\left(\phi^{1}\left(z_{1}, z_{2}\right)\right.$, $\left.\phi^{2}\left(z_{1}, z_{2}\right)\right)$, then $\alpha z_{1}+\beta z_{2}=0$ implies that $\alpha \phi^{1}\left(z_{1}, z_{2}\right)+\beta \phi^{2}\left(z_{1}, z_{2}\right)=0$; i.e. $\alpha \phi^{1}\left(z_{1},-\alpha / \beta z_{1}\right)+$ $\beta \phi^{2}\left(z_{1},-\alpha / \beta z_{1}\right)=0$ for all $z_{1} \in \mathbf{R}$. Thus

$$
\left.\alpha \frac{\partial \phi^{1}}{\partial z_{1}}-\frac{\alpha}{\beta} \frac{\partial \phi^{1}}{\partial z_{2}}\right)+\beta\left(\frac{\partial \phi^{2}}{\partial z_{1}}-\frac{\alpha}{\beta} \frac{\partial \phi^{2}}{\partial z_{2}}\right)=0 .
$$

Hence

$$
\begin{aligned}
\mathrm{d} \phi(\gamma) h= & h_{1}\left[\frac{\partial \phi^{1}}{\partial z_{1}} \frac{\alpha}{\beta}-\frac{\partial \phi^{1}}{\partial z_{2}}, \frac{\partial \phi^{2}}{\partial z_{1}}-\frac{\alpha}{\beta} \frac{\partial \phi^{2}}{\partial z_{2}}\right]^{t} \\
& =h_{1}\left[\frac{\partial \phi^{1}}{\partial z_{1}}-\frac{\alpha}{\beta} \frac{\partial \phi^{1}}{\partial z_{2}}, \frac{-\alpha}{\beta}\left(\frac{\partial \phi^{1}}{\partial z_{1}}-\frac{\alpha}{\beta} \frac{\partial \phi^{1}}{\partial z_{2}}\right)\right]^{t},
\end{aligned}
$$

is of the desired form. If $\beta=0$, a similar proof works. We then have

THeOrem 2. Suppose that $\phi$ locally conjugates $X_{\sigma}$ to $X_{\tau}$ and leaves the lines $L_{i}$ invariant, $i=1,2$. If $\gamma$ is a strongly non-degenerate flow of $X_{t}$ satisfying (3), then $\phi(\gamma)$ is a strongly non-degenerate flow of $X$ satisfying (3). 
We shall illustrate the theorem by explicitly constructing the local conjugations for the system (1), with homogeneous Neumann boundary conditions

$$
u^{\prime}(a)=u^{\prime}(b)=0 \text {. }
$$

Here

$$
X_{s}(u, v)=\left(v,-\frac{1}{s} f(u)\right)
$$

and we assume $f(-A)=f(B)=f(0)=0, A, B>0, f^{\prime}(0)>0$ and for $u \neq 0$,

$$
f(u) / u>f^{\prime}(u),-A \leqslant u \leqslant B .
$$

The phase portrait for the flow can be depicted as in Fig. 2. Observe that solutions of (1), (8) correspond to orbits which meet the $u$-axis at $x=a$ and $x=b$, and hence must lie in the "tearshaped" region $R_{s}$ corresponding to $X_{s}$ in Fig. 2; we shall construct all of our maps in the interior of $R_{s}$ minus the origin.

For any point $P=(p, 0), p<0$ in $R_{s}, P$ is carried by the flow to a unique point $P^{\prime}=\left(p^{\prime}, 0\right)$, $p^{\prime}>0$; let $T(s, p)$ denote the corresponding (shortest) "time". Since $F\left(p^{\prime}\right)=F(p)$, where $F^{\prime}=f$,

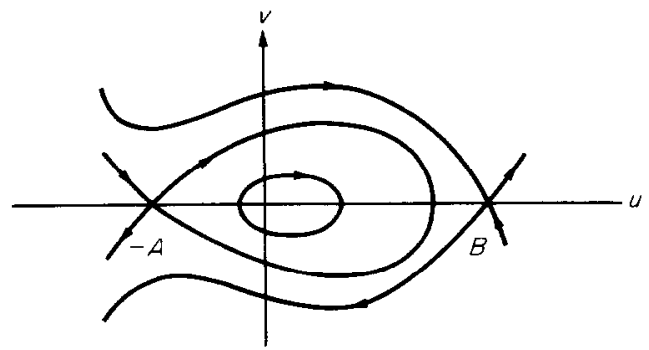

Figure 2

it follows that $p^{\prime}$ is independent of $s$. Hence since

$$
T(s, p)=\sqrt{\frac{s}{2}} \int_{0}^{p^{\prime}}\left(F\left(p^{\prime}\right)-F(p)\right)^{-1 / 2} \mathrm{~d} u,
$$

we see that $T$ is an increasing function of $s$. Furthermore, (10) implies that $T$ is a decreasing function of $p$. These remarks allow us to construct the desired conjugations; first we need some notation. For $z \in R_{s}$, and $W$ a point on the orbit of $X_{s}$ through $Z$, define $\tau_{s}(Z, W)$ to be the (shortest) time that the orbit takes to go from $Z$ to $W$; thus $\tau_{s}\left(P, P^{\prime}\right)=T(s, p)$, where $P$ and $\mathrm{P}^{\prime}$ are as above.

Now let $t<s$, and let $P \in R_{s}$ be as before. We set $\phi_{1}(P)=Q$ where $Q=(q, 0) \in R_{t}$ and $T(s, P)=$ $T(t, Q)$. We extend $\phi_{1}$ to all of $R_{s}$ by the flow in the natural way, and call this extension $\phi$. Thus, if $Z \in R_{s}$, we set $\phi(Z)=W$, where $\tau_{s}(P, Z)=\tau_{t}(Q, W)$, and $\phi_{1}(P)=Q$. It is easy to check that $\phi_{1}$ is well defined; we must show that $\phi$ is well-defined, i.e. that $\phi$ and $\phi_{1}$ agree on their common domain. Let $P=(p, 0) \varepsilon R_{s}, p<0$, and $\phi(P)=\tilde{Q}$. If $Q \neq \tilde{Q}$, say e.g. $T_{t}(Q, \widetilde{Q})<T_{t}(Q, Q)$. Then by symmetry about the $u$-axis, $2 T_{s}\left(P, P^{\prime}\right)=T_{s}(P, P)=T_{t}(Q, \widetilde{Q})=T_{t}\left(Q, Q^{\prime}\right)+T_{t}(Q, \widetilde{Q})$, so $T_{s}\left(P, P^{\prime}\right)$ $=T_{t}\left(Q, Q^{\prime}\right)<T_{t}\left(Q, Q^{\prime}\right)$, a contradiction. We similarly get a contradiction if $T_{t}(Q, \widetilde{Q})>T_{t}(Q, Q)$. Thus $\phi$ is well-defined. Clearly $\phi$ is a local diffeomorphism which leaves the $u$-axis invariant. Thus, we have:

THEOREM 3. Suppose (10) holds, and $X_{s}, X_{t}, t<s$ are vector fields defined by (9). Then there is a local conjugation of $X_{s}$ into $X_{t}$ which leaves $\{v=0\} \cap R_{s}$ invariant. In particular all flows of $X_{s}$ satisfying (8) are strongly non-degenerate. 
The last sentence follows from [3, p. 78], where it is shown that all flows of $X_{s}$ having sufficiently small norm are strongly non-degenerate.

Observe now that this technique does not work so nicely for other types of boundary conditions since we strongly used the symmetry of the flows about the $u$-axis. This difficulty can be remedied, however, by "lifting" the flows to the universal covering space.

\section{A COVERING SPACE APPROACH}

Rather than develop a complete theory, we shall content ourselves with discussing the Dirichlet problem for (1); the reader should find no difficulty in considering more general boundary conditions and general vector fields. Thus, we consider flows of (9), together with homogeneous boundary conditions $\S$

$$
u(a)=u(b)=0 \text {. }
$$

Referring to Fig. 2, for solutions which have one internal maximum (equivalently, orbits which begin and end on the $v$-axis, and do not encircle the origin, in Fig. 2), we can argue as in section 3 , and construct conjugations in the regions $R_{s} \cap\{u \leqslant 0\}$, and $R_{s} \cap\{u \geqslant 0\}$. For the other cases, where the orbits encircle the origin, we use covering spaces to "unwind" the orbits.

For the vector field $X_{s}$, defined by (9), and under the same assumptions on $f$ as in section 3 , we let $R_{s}$ be as in Section 3, and let $\left(0, a_{s}\right)=R_{s} \cap\{u \geqslant 0\}$. Let $\bar{R}_{s}$ be the "universal cover" of $R_{s}$, i.e. $\bar{R}_{s}=\mathbf{R} \times\left(0, a_{s}\right)$. We fix an integer $k>1$, and for $0<p<a_{s}$, we define $T_{s}^{k}(p)$ to be the "time" that the orbit of $X_{s}$, through $(0, p)$ takes to make $k \pi$ revolutions about the origin. For the convenience of the reader, we shall now outline our strategy. We first construct a local diffeomorphism $\pi: \bar{R}_{s} \rightarrow R_{s}$, which will allow us to "lift" the vector field $X_{s}$ to a vector field $\bar{X}_{s}$ on $\bar{R}_{s}$, defined by $\mathrm{d} \pi\left(\bar{X}_{s}(P)\right)=X_{s}(\pi(P)), P \in \bar{R}_{s}$. This map carries orbits of $\bar{X}_{s}$ into orbits of $X_{s}$ (if $\bar{\sigma}^{\prime}=\bar{X}_{s}(\bar{\sigma})$, then $\pi(\bar{\sigma})^{\prime}=\mathrm{d} \pi(\bar{\sigma}) \bar{\sigma}^{\prime}=\mathrm{d} \pi(\bar{\sigma}) \bar{X}_{s}(\bar{\sigma})=X_{s}(\pi(\bar{\sigma}))$ and if $\bar{\sigma}(a)=0$, then $\left.\pi(\bar{\sigma}(a))=0\right)$ and every orbit "downstairs" lifts uniquely to one "upstairs", provided that we specify $\bar{\sigma}(a)$, say $\bar{\sigma}(a)=0$ (but not conversely since orbits upstairs whose second components are fixed, and whose first components differ by an integral multiple of $k \pi$ map to the same orbits downstairs). Our analysis will take place on the orbits of $\bar{X}_{s}$, which take a particularly simple form; this will enable us to make conclusions about orbits of $X_{s}$. We proceed now with the details.

Let $0<p<a_{s}$, and denote by $\gamma(t, p)$ the orbit of $X_{s}$ through $(0, p)$ (the dependence on $s$ will be suppressed), and set

$$
\pi(t, p)=\gamma\left(\frac{t T_{s}^{k}(p)}{k \pi}, p\right) .
$$

Note $\pi(0, p)=p$, and if $k$ is odd $\pi(k \pi, p)=-p$, while $\pi(k \pi, p)=p$ if $k$ is even, and that the curve $\mathbf{R} \times p$ (a straight line in $\left.\bar{R}_{s}\right)$, projects to an orbit in $R_{s}$. Thus by what we have said above, this line is an orbit of $\bar{X}_{s}$, and so by uniqueness, these are all the orbits of $\bar{X}_{s}$; i.e. all orbits of $\bar{X}_{s}$ are straight lines.

Suppose now

$$
\begin{aligned}
& \gamma\left(\frac{t T_{s}^{k}(p)}{k \pi}, p\right)=\gamma\left(\frac{t^{\prime} T_{s}^{k}(q)}{k \pi}, q\right), \\
& 0<p, \quad q<a_{s^{\prime}}
\end{aligned}
$$

$\S$ e.g., boundary conditions of the form $\alpha u(a)+\beta u^{\prime}(a)=\alpha u(b)+\beta u^{\prime}(b)=0$. 
Then $p=q$, so that $\left(t-t^{\prime}\right) T_{s}^{k}(p)=k \pi(2 n \pi), n \in \mathbf{Z}$. Thus if $\left|t-t^{\prime}\right|<2 k \pi^{2} / T_{s}^{k}(p)$, then $(t, p)$ and $\left(t^{\prime}, q\right)$ map into different points; i.e., $\pi(t, p) \neq \pi\left(t^{\prime}, q\right)$ and so $\pi$ is locally one-one. To see that $\mathrm{d} \pi$ is locally one-one, we note that $\pi=\delta \cdot \theta$, where $\theta$ and $\delta$ are defined by

$$
(t, p) \stackrel{\theta}{\rightarrow}\left(\frac{t T_{s}^{k}(p)}{k \pi}, p\right) \stackrel{\delta}{\rightarrow} \gamma\left(\frac{t T_{s}^{k}(p)}{k \pi}, p\right) .
$$

Now from general considerations, the map $(t, q) \rightarrow \gamma(t, q)$ is a local diffeomorphism, while a straightforward computation, (assuming $\partial T_{s}^{k}(p) / \partial p \neq 0$ ) shows that $\mathrm{d} \theta$ is non-singular. It follows that $\pi$ is a local diffeomorphism.

Next, we shall show that

$$
\bar{X}_{s}(t, p)=\left(k \pi / T_{s}^{k}(p), 0\right)
$$

First note that since $\mathrm{d} \pi \bar{X}_{s}=X_{s}$, and orbits in $\bar{R}_{s}$ are straight lines, the second component of $\bar{X}_{s}$ is zero. Thus, we seek $\lambda \in \mathbf{R}$ such that $\mathrm{d} \pi(\lambda, 0)^{t}=X_{s}$. Using (12) we get $X_{s}=\mathrm{d} \pi(\lambda, 0)^{t}=\mathrm{d} \delta \mathrm{d} \theta(\lambda, 0)^{t}$ $=\mathrm{d} \delta\left(\lambda T_{s}^{k}(p) / k \pi, 0\right)^{t}=\left(X_{s}, \gamma_{p}\right)\left(\lambda T_{s}^{k}(p) / k \pi, 0\right)^{t}=\left(\lambda T_{s}^{k}(p) / k \pi\right) X_{s^{\prime}}$. Hence $\lambda=k \pi / T_{s}^{k}(p)$, and (13) holds.

Next, let $\bar{\gamma}^{\prime}=\bar{X}_{s}(\bar{\gamma})$; we show $\bar{\gamma}$ is strongly non-degenerate. Thus suppose $\bar{h}^{\prime}=\mathrm{d} \bar{X}_{s}(\bar{\gamma}) \bar{h}$, where $\bar{h}=\left(\bar{h}_{1}, \bar{h}_{2}\right)$, and $\bar{h}_{1}(a)=\bar{h}_{1}(b)=0$. Since

$$
\left(\frac{\bar{h}_{1}}{\bar{h}_{2}}\right)=-k \pi T_{s}^{k}(p)^{-2} \frac{\partial T_{s}^{k}(p)}{\partial p}\left(\begin{array}{ll}
0 & 1 \\
0 & 0
\end{array}\right)\left(\frac{h_{1}}{\bar{h}_{2}}\right),
$$

we see that $\bar{h}_{2}^{\prime}=0$, so $\bar{h}_{2}=c$, a constant. Also

$$
\bar{h}_{1}^{\prime}=-c k \pi T_{s}^{k}(p)^{-2} \frac{\partial T_{s}^{k}(p)}{\partial p}=d,
$$

a constant. Thus $h_{1}=\mathrm{d} t+\mathrm{e}$, and using the boundary conditions gives $0=\mathrm{d} a=\mathrm{e}$, and $0=\mathrm{d} b+\mathrm{e}$, so $d=\mathrm{e}=0$ and $\bar{h}_{1}=0$. Hence $d=\bar{h}_{1}^{\prime}=0$ so $c=0$, and $\bar{h}=0$.

To finish the proof, we must show that if $\bar{\gamma}$ is a flow of $\bar{X}_{s}$ which satisfies the boundary conditions, then $\pi(\bar{\gamma})$ is also strongly non-degenerate. First note that if $\bar{\gamma}(t)=\left(\gamma_{1}(t), p\right)$, then if $\bar{\gamma}$ satisfies the boundary conditions, $\gamma_{1}(a)=0, \gamma_{1}(b)=k \pi$, and from (12)

$$
(\pi \bar{\gamma})(t)=\pi\left(\gamma_{1}(t), p\right)=\gamma\left(\gamma_{1}(t) T_{s}^{k}(p)(k \pi)^{-1}, p\right)
$$

so $(\pi \bar{\gamma})(a)=\gamma(0, p)=p$, and $(\pi \bar{\gamma})(b)-\gamma\left(T_{s}^{k}(p), p\right)= \pm p$, depending on whether $k$ is even or odd. Hence $\pi \bar{\gamma}$ satisfies the correct boundary conditions if $\bar{\gamma}$ does. We can now show that $\pi(\bar{\gamma})$ is strongly non-degenerate.

Now if $h$ is sufficiently small, then $\pi(\bar{\gamma})+h$ lifts uniquely to $\bar{\gamma}+\bar{h}$ for some $h$. Thus suppose $\mathrm{d} Y_{s}(\pi(\bar{\gamma})) h=0$, where $h_{1}(a)=h_{1}(b)=0$. Then if $\theta=t h, t>0, t$ small, $\mathrm{d} Y_{s}(\pi(\bar{\gamma})) k=0$ and $\theta(a)=\theta(b)=0$. To compute $\mathrm{d} Y_{s}(\pi(\bar{\gamma})) \theta$, we compute the linear parts (1.p.): For sufficiently small $t$, we have

$$
\begin{aligned}
0=\mathrm{d} Y_{s}(\pi(\bar{\gamma})) \theta & =1 . p \cdot\left[Y_{s}(\pi(\bar{\gamma})+\theta)-Y_{s}(\pi(\bar{\gamma}))\right] \\
& =1 . p .\left[Y_{s}(\pi(\bar{\gamma}+\bar{\theta}))-Y_{s}(\pi(\bar{\gamma}))\right] \\
& =1 . p .\left[\mathrm{d} \pi\left(Y_{s}(\bar{\gamma}+\bar{\theta})\right)-\pi\left(Y_{s}(\bar{\gamma})\right)\right] \\
& =\mathrm{d} \pi \mathrm{d} Y_{s}(\bar{\gamma}) \bar{\theta} .
\end{aligned}
$$

Now since $\bar{\gamma}$ is strongly non-degenerate $\bar{\theta}=0$, so $\pi(\bar{\gamma})+\theta=\pi(\bar{\gamma}+\bar{\theta})=\pi(\bar{\gamma})$, so $\theta=0$ and thus 
$h=0$, and $\pi(\bar{\gamma})$ is strongly non-degenerate. Since $\pi$ is a local diffeomorphism, it follows that all flows of (1) satisfying (11) are strongly non-degenerate.

Finally, we note that the vector fields $\bar{X}_{s}$ and $\bar{X}_{t}, t<s$, are always local conjugates at one another. To see this, define a map $F: \bar{R}_{s} \rightarrow \bar{R}_{t}$ by $F(\theta, p)=(\theta, \psi(p))$, where $T_{s}^{k}(p)=T_{s}^{k}(\psi(p))$. Then $F$ is a local diffeomorphism and

$$
\begin{aligned}
\mathrm{d} F \bar{X}_{s}(\theta, p) & =\left[\begin{array}{ll}
1 & 0 \\
0 & \mathrm{~d} \psi
\end{array}\right]\left(\begin{array}{c}
k \pi / T_{s}^{k}(p) \\
0
\end{array}\right)=\left(\begin{array}{c}
k \pi / T_{s}^{k}(p) \\
0
\end{array}\right) \\
& =\left(\begin{array}{c}
k \pi / T_{\mathrm{t}}^{k}(\psi(p)) \\
0
\end{array}\right)=\bar{X}_{t}(\theta, \psi(p)) \\
& =\bar{X}_{t}(F(\theta, p)) .
\end{aligned}
$$

We remark that we have shown that if $\partial T_{s}^{k} / \partial p \neq 0$, then solutions of (2)-(3) are strongly nondegenerate. But we have remarked earlier (after the proof of Theorem 1) that under the hypotheses of theorem 1 , that $\partial T_{s}^{k} \partial p \neq 0$. It follows then, that we have the following strengthening of Theorem 1.

TheOREM 4. Under the hypotheses of Theorem 1, all solutions of (2) satisfying the boundary conditions $\alpha u(a)+\beta v(a)=\alpha u(b)+\beta u(b)=0$, are strongly non-degenerate.

We of course also obtain the analogue of Corollary 1 for strongly non-degenerate solutions.

\section{SOME FINAL REMARKS}

We have only considered orbits which satisfy (4), or more precisely, $X_{\sigma_{t}(p)} \cdot \sigma_{t(p)} \neq 0$, for all $t \in[a, b]$. However, we may also allow $X_{a b(p)} \cdot \sigma_{t}(p)=0$, since the limit of monotone functions is also monotone; in this case $T$ is defined only on a half-open interval. If $X_{\sigma a(p)} \cdot \sigma_{a}(p)=0$, there is gain no problem. If both are zero, then the intersection of the half-open intervals may be a point.

Internal tangencies may be classified into two sets, good and bad. (See Fig. 3).

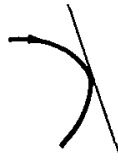

Good

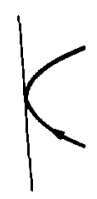

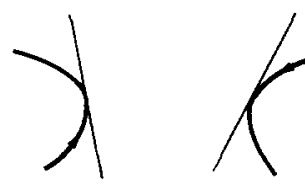

Bad

Figure 3

It is easy to compute $d \sigma_{t}(p)$ for small $|t|$ near the tangency and thus see that good internal tangencies do not produce bifurcations if $\tilde{p} \mathrm{~d} X_{p} \mathrm{p}-X_{p} \cdot \tilde{p} \geqslant 0$, while bad tangencies always produce bifurcations if $b-a$ is small. A complete study of this phenomenon seems to require a condition on the curvature of the orbit, and would involve higher derivatives of $X$. 


\section{REFERENCES}

1. Chafee, N., Asymptotic behaviour for solutions of a one-dimensional parabolic equation with homogeneous Neumann boundary conditions, J. diff. Equns 18, 111-135 (1975).

2. Hirsch, M. \& Smale, S., Differential Equations, Dynamical Systems, and Linear Algebra. Academic Press, New York (1974).

3. NireNberg, L., Nonlinear Functional Analysis. C.I.M.S. Lecture Notes, (1973-1974). 